\title{
SISTEMA PARA LOCALIZAÇÃO DE FALTAS EM LINHAS DE TRANSMISSÃO COM SUBESTAÇÕES CONECTADAS EM DERIVAÇÃO
}

\author{
Giovanni Manassero Junior* \\ giomanjr@pea.usp.br \\ Eduardo Cesar Senger* \\ sengerapea.usp.br
}

\author{
Emílio César Neves Rodrigues ${ }^{\dagger}$ \\ ecrodriguesdcteep.com.br
}

\author{
Renato Mikio Nakagomi*
}

rmikio@pea.usp.br

\author{
Eduardo Lorenzetti Pellini* \\ epellini@pea.usp.br
}

*Av. Prof. Luciano Gualberto, 158 - travessa 3 - sala A2-14

CEP: 05508-900 - São Paulo, SP, Brasil

${ }^{\dagger}$ Alameda CESP, $\mathrm{s} / \mathrm{n}^{\circ}$

CEP: 13202-970 Jundiaí, SP, Brasil

\begin{abstract}
Fault Location System for Multi-terminal Transmission Lines

This paper presents the development and implementation of algorithms for fault location in multi-terminal transmission lines. The proposed fault location system is capable of correctly identifying the fault point based on voltage and current phase components, calculated using measurements of voltage and current signals from intelligent electronic devices, located on the transmission line terminals. The algorithms, which are parte of the system, have access to the electrical parameters of the transmission lines and to information about the transformers loading and their connection type. This work also presents the development of phase component models for the power system elements used by the fault location algorithms.
\end{abstract}

KEYWORDS: Fault location, Power transmission lines, Substations.

Artigo submetido em 04/11/2008 (Id.: 00916)

Revisado em 26/01/2009, 12/08/2009

Aceito sob recomendação do Editor Associado Prof. Julio Cesar Stacchini Souza

\section{RESUMO}

Este trabalho tem por objetivo apresentar o desenvolvimento e a implementação de algoritmos para a localização de faltas em linhas de transmissão com subestações conectadas em derivação. O sistema proposto é capaz de identificar corretamente o ponto de ocorrência do defeito utilizando as componentes de fase dos fasores de tensões e correntes, calculadas através dos registros desses sinais, efetuados por oscilógrafos digitais e/ou relés de proteção instalados nos terminais da linha de transmissão. Os algoritmos que integram este sistema têm acesso aos parâmetros elétricos da linha e informações sobre o tipo de ligação e o carregamento dos transformadores conectados aos terminais das derivações. Este trabalho apresenta também o desenvolvimento dos modelos para os elementos do sistema de transmissão, em componentes de fase. Estes modelos são utilizados pelos algoritmos que integram a metodologia para localização de faltas.

PALAVRAS-CHAVE: Proteção de sistemas elétricos, Linhas de transmissão de energia elétrica, Subestações elétricas 


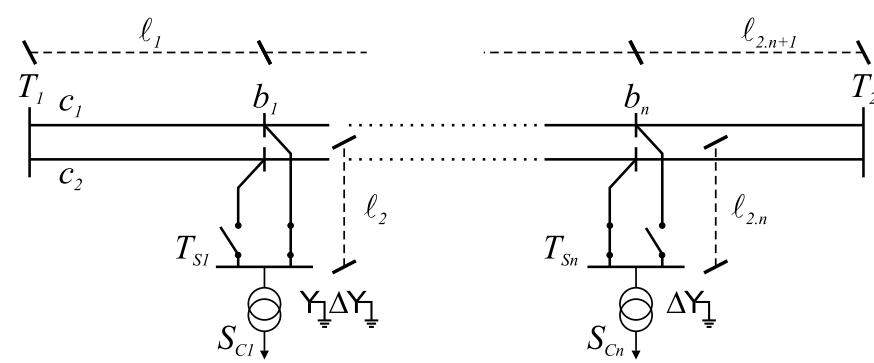

Figura 1: Sistema de transmissão.

\section{NOMENCLATURA}

ATP: Alternative Transient Program

GPS: Global Positioning System

\section{INTRODUÇÃO}

Linhas de transmissão são responsáveis pela interligação entre os centros de geração e os centros de consumo e podem ser classificadas de acordo com o nível de tensão em que operam.

No nível de tensão de subtransmissão é comum a existência de uma ou mais subestações conectadas em derivação nas linhas de transmissão. Quando ocorre um defeito permanente em uma destas linhas, o trabalho das equipes de emergência para localizar o ponto de falta é habitualmente mais demorado, uma vez que não se sabe exatamente qual o trecho onde o defeito ocorreu. Isto implica no aumento dos custos de manutenção e na redução da confiabilidade do sistema.

A desregulamentação do setor elétrico contribuiu para o aumento na complexidade do problema, visto que, normalmente, as subestações conectadas em derivação não são de propriedade das concessionárias de transmissão e, por razões comerciais, as informações disponíveis nos terminais dessas subestações (registros de oscilografia, medições de tensões e correntes de pré-falta, etc.), que permitiriam melhorar o processo de localização das faltas, nem sempre são compartilhadas com a empresa responsável pelo sistema.

\subsection{Sistema de Transmissão}

A Fig. 1 ilustra a configuração típica das redes consideradas neste trabalho. Esta rede é constituída por uma linha de transmissão, denominada tronco, que interliga duas subestações de propriedade da concessionária de transmissão $\left(T_{1}\right.$ e $\left.T_{2}\right)$. Ao longo do tronco há pontos de derivação $\left(b_{1}\right.$ a $\left.b_{n}\right)$ de onde partem linhas de transmissão, que alimentam subestações de propriedade de consumidores primários ou de concessionárias de distribuição $\left(T_{S 1}\right.$ a $\left.T_{S n}\right)$.
Este sistema pode ser constituído por linhas de circuito sim$T$, ples ou duplo. Desta forma, o tronco pode ser constituído por uma única linha de circuito simples; uma linha de circuito duplo (dois circuitos na mesma torre); duas linhas de circuito simples instaladas na mesma faixa de passagem ou em faixas de passagem distintas. No caso de redes de circuito duplo, as subestações dos consumidores estão conectadas a apenas um dos circuitos através de uma chave seccionadora.

Nos terminais $T_{1}$ e $T_{2}$ há equipamentos responsáveis pelo registro dos sinais de tensões e correntes, que podem ou não estar sincronizados temporalmente via GPS. Entretanto, nos terminais $T_{S 1}$ a $T_{S n}$ considera-se que estes registros podem não estar disponíveis. No entanto, o sistema proposto é capaz de localizar corretamente o ponto da falta, utilizando apenas os dados dos terminais $T_{1}$ e $T_{2}$. Caso existam dados nos demais terminais, eles são utilizados para aumentar a precisão dos resultados.

A inexistência de registros em uma ou mais subestações implica que a carga existente nessas subestações é desconhecida. Contudo, o sistema proposto dispõe de um método capaz de estimar o seu valor. Para tanto, adota-se a hipótese de que a carga não contribui com a corrente de falta, isto é, não existe geração distribuída em paralelo com as cargas. Porém, é importante salientar que nos transformadores dessas subestações pode-se empregar a ligação estrelaaterrada/delta/estrela-aterrada. Isto implica que uma parcela significativa da corrente de falta, para defeitos que envolvem a terra, pode retornar através do enrolamento primário destes transformadores. Este cenário torna o problema de localização da falta bastante complexo, já que uma parcela considerável da corrente de curto-circuito pode ser injetada por este tipo de transformador. No caso das subestações em que os registros de tensões e correntes não estão disponíveis, o valor desta corrente não é um dado de entrada para o sistema de localização, entretanto, o modelo matemático utilizado na representação destes transformadores considera, implicitamente, o retorno dessa parcela da corrente de falta.

\subsection{Revisão da Literatura}

No levantamento da literatura existente, foram encontrados algoritmos bem consolidados para a localização de faltas em linhas de transmissão com apenas uma derivação, (Aggarwal et al., 1993; Girgis et al., 1992; Yu et al., 2001; Tziouvaras et al., 2001; Lin et al., 2002; Lai et al., 2000). Entretanto, para linhas de transmissão com várias subestações conectadas em derivação há poucas publicações, (Abe et al., 1995; Brahma, 2005; Nagasawa et al., 1992; Funabashi et al., 2000). Além disso, estas publicações não atendem a todos os requisitos descritos no item 2 .

Os métodos apresentados em (Abe et al., 1995) e (Brahma, 


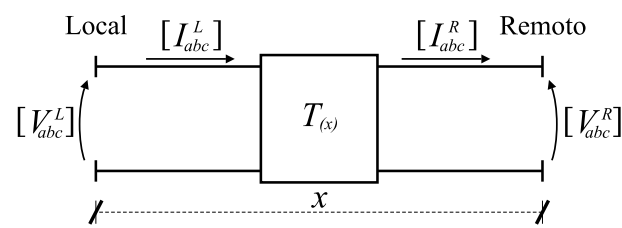

Figura 2: Trecho de linha - Modelo de quadripolos de transmissão

2005) se limitam à localização em linhas de circuito simples, a partir dos fasores de tensões e correntes em todos os seus terminais, sincronizados entre si. Estes métodos utilizam o modelo de linha curta e, desta forma, têm sua precisão afetada quando aplicados a linhas médias e longas.

O métodos descritos em (Nagasawa et al., 1992) e (Funabashi et al., 2000) foram desenvolvidos para localização em linhas de circuito duplo e também utilizam o modelo de linha curta. O primeiro é baseado nas componentes simétricas dos fasores das correntes, em todos os terminais da rede, porém não fica claro se o método exige sincronização entre as medições, além disso, as hipóteses adotadas são pouco usuais e de difícil verificação. O segundo apresenta dois algoritmos para localização de faltas que se baseiam nos fasores das tensões e correntes em todos os terminais da linha, sincronizados entre si.

Por estas razões, o método proposto neste trabalho e apresentado nos próximos capítulos, pretende contribuir para o tema, apresentando uma solução que seja suficientemente precisa e ao mesmo tempo adequada em termos de exigência dos dados de entrada, de forma a atender a realidade encontrada nas empresas de transmissão.

\section{MODELAMENTO DOS COMPONENTES DO SISTEMA}

De forma a aumentar a precisão dos resultados, o sistema proposto neste trabalho consiste no equacionamento trifásico da rede de transmissão. Para tanto, foram desenvolvidos modelos matemáticos bastante precisos dos componentes que constituem a rede elétrica (linhas de transmissão, transformadores, cargas das subestações conectadas em derivação e os curtos-circuitos).

Estes modelos estão apresentados nos itens a seguir. Vale ressaltar que a representação das linhas de transmissão em componentes de fase considera a assimetria, o efeito das capacitâncias e eventuais pontos de transposição.

\subsection{Linhas de transmissão}

A Fig. 2 ilustra uma linha de transmissão de circuito simples e comprimento $x$, representada pelo modelo de quadripolos de transmissão, em componentes de fase. Esta linha é caracterizada pelas matrizes de impedâncias série $\left[z_{a b c}\right]$ e admitâncias paralela $\left[y_{a b c}\right]$, distribuídas uniformemente ao longo do seu comprimento. A equação (1) descreve a relação entre as tensões e correntes em ambos os terminais desta linha.

$$
\left[\begin{array}{l}
V_{a b c}^{L} \\
I_{a b c}^{L}
\end{array}\right]=\left[T_{(x)}\right] \times\left[\begin{array}{c}
V_{a b c}^{R} \\
I_{a b c}^{R}
\end{array}\right] \text { e }\left[\begin{array}{c}
V_{a b c}^{R} \\
I_{a b c}^{R}
\end{array}\right]=\left[T_{(x)}\right]^{-1} \times\left[\begin{array}{c}
V_{a b c}^{L} \\
I_{a b c}^{L}
\end{array}\right]
$$

Onde a matriz de parâmetros de transmissão $\left[T_{(x)}\right]$ é dada pela equação (2) e os parâmetros do quadripolo de transmissão podem ser obtidos através do sistema (3), conforme a referência (Bowman and McNamee, 1964).

$$
\begin{gathered}
{\left[T_{(x)}\right]=\left[\begin{array}{ll}
{\left[A_{(x)}\right]} & {\left[B_{(x)}\right]} \\
{\left[C_{(x)}\right]} & {\left[D_{(x)}\right]}
\end{array}\right]} \\
{\left[A_{(x)}\right]=\left[y_{a b c}\right]^{-1} \times[M] \times\left\{\left[\cosh \left(\gamma_{j} \cdot x\right)\right]\right\} \times[M]^{-1} \times\left[y_{a b c}\right]} \\
{\left[B_{(x)}\right]=\left[y_{a b c}\right]^{-1} \times[M] \times\left\{\gamma_{j} \cdot\left[\sinh \left(\gamma_{j} \cdot x\right)\right]\right\} \times[M]^{-1}} \\
{\left[C_{(x)}\right]=x \cdot[M] \times\left\{\left[\frac{\sinh \left(\gamma_{j} \cdot x\right)}{\left(\gamma_{j} \cdot x\right)}\right]\right\} \times[M]^{-1} \times\left[y_{a b c}\right]} \\
{\left[D_{(x)}\right]=[M] \times\left\{\left[\cosh \left(\gamma_{j} \cdot x\right)\right]\right\} \times[M]^{-1}}
\end{gathered}
$$

Onde:

$[M]$ : autovetores da matriz $[P]=\left[y_{a b c}\right] \times\left[z_{a b c}\right]$;

$\gamma_{j}^{2}$ : autovalores da matriz $[P]$;

$\{[]\}$ : notação para matriz diagonal.

Estes parâmetros podem ser obtidos para qualquer arranjo de linhas de transmissão (circuito simples ou duplo). Para linhas de circuito duplo, é preciso observar o acoplamento entre os circuitos e, para o caso de linhas transpostas, são utilizadas matrizes $\left[z_{a b c}\right]$ e $\left[y_{a b c}\right]$ distintas, para cada trecho entre os pontos de transposição.

\subsection{Conjunto carga e transformador}

Para o desenvolvimento do modelo das cargas em componentes de fase, foram adotadas as seguintes hipóteses: ausência de geração nas subestações conectadas em derivação, isto é, a carga alimentada pelo transformador conectado ao terminal da subestação não contribui com a corrente de curto-circuito; e a carga é modelada por impedância constante, equilibrada e ligada em delta.

Para o desenvolvimento dos modelos dos transformadores das subestações conectadas em derivação, em componentes de fase, foram adotados os diagramas seqüenciais para 


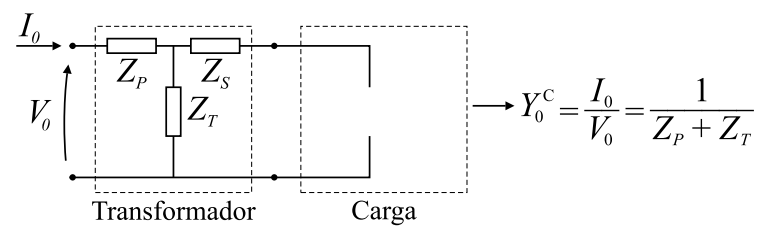

(a) seqüência zero

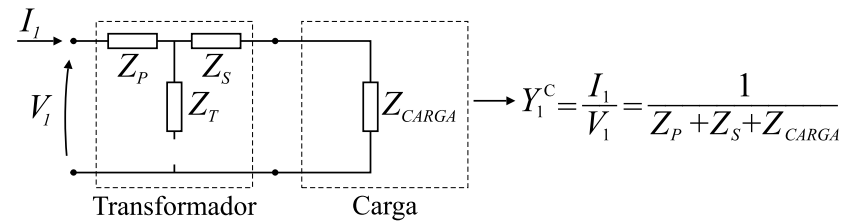

(a) seqüência positiva

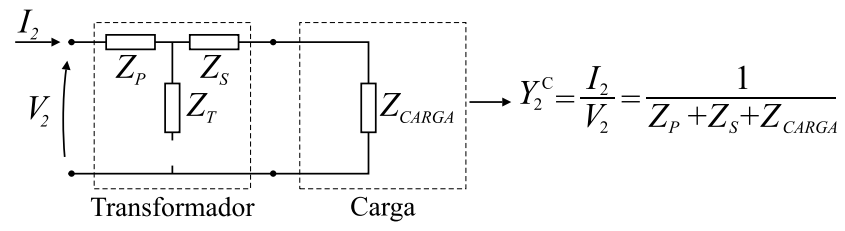

(a) seqüência negativa

Figura 3: Diagramas seqüenciais do conjunto carga e transformador

cada tipo de ligação dos seus enrolamentos (Ramos and Dias, 1983). Desta forma, para a obtenção do modelo do conjunto carga e transformador é preciso associar as admitâncias de ambos, em componentes simétricas, e efetuar as transformações adequadas na matriz resultante desta associação. A Fig. 3 ilustra os diagramas seqüenciais para o conjunto carga e transformador, considerando a ligação estrelaaterrada/delta/estrela-aterrada.

A relação entre os fasores das tensões e correntes no primário do transformador apresentado na Fig. 3, em componentes simétricas, pode ser escrita conforme em (4).

$$
\left[I_{012}\right]=\left[\begin{array}{ccc}
Y_{0}^{C} & 0 & 0 \\
0 & Y_{1}^{C} & 0 \\
0 & 0 & Y_{2}^{C}
\end{array}\right] \times\left[V_{012}\right]=\left[Y_{012}^{C}\right] \times\left[V_{012}\right]
$$

Entretanto, a relação entre tensões e correntes, em componentes simétricas, e tensões e correntes, em componentes de fase, pode ser dadas pelas equações (5) e (6), onde $\left[T_{\text {sim }}\right]$ é a matriz de transformação de componentes simétricas:

$$
\begin{aligned}
& {\left[V_{012}\right]=\left[T_{\text {sim }}\right]^{-1} \times\left[V_{a b c}\right]} \\
& {\left[I_{012}\right]=\left[T_{\text {sim }}\right]^{-1} \times\left[I_{a b c}\right]}
\end{aligned}
$$

Portanto, a equação (4), ser escrita conforme (7):

$$
\left[T_{s i m}\right]^{-1} \times\left[I_{a b c}\right]=\left[Y_{012}^{C}\right] \times\left[T_{s i m}\right]^{-1} \times\left[V_{a b c}\right]
$$

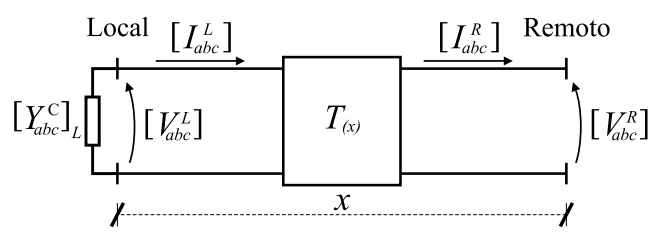

Figura 4: Trecho de linha - Cálculo da matriz $\left[Y_{a b c}^{e q}\right]_{x}$

Onde:

$$
\left[T_{s i m}\right] \times\left[Y_{012}^{C}\right] \times\left[T_{s i m}\right]^{-1}=\left[Y_{a b c}^{C}\right]
$$

\subsection{Associação entre o trecho de linha e o conjunto carga e transformador}

Além da matriz de admitâncias do conjunto carga e transformador, o sistema proposto utiliza uma matriz equivalente que é resultado da associação entre transformador, carga e trecho de linha. Esta matriz é obtida através da relação entre tensões e correntes, no terminal do trecho de linha que alimenta a carga e o transformador e, portanto, depende da matriz de admitâncias da associação carga e transformador, e da matriz do quadripolo de transmissão do trecho de linha em questão.

A Fig. 4 ilustra um trecho de linha de comprimento $x$, compreendido entre os terminais local e remoto. As tensões e correntes no terminal remoto deste trecho podem ser obtidas a partir de (1), conforme detalhado em (9).

$$
\left[\begin{array}{c}
V_{a b c}^{R} \\
-I_{a b c}^{R}
\end{array}\right]=\left[\begin{array}{l}
{\left[A_{(x)}\right.} \\
{\left[C_{(x)}\right.}
\end{array}\right] \quad\left[\begin{array}{c}
\left.B_{(x)}\right] \\
\left.D_{(x)}\right]
\end{array}\right] \times\left[\begin{array}{c}
V_{a b c}^{L} \\
-I_{a b c}^{L}
\end{array}\right]
$$

Reescrevendo (7), que representa o modelo do conjunto carga e transformador, resulta em:

$$
-\left[I_{a b c}^{L}\right]=\left[Y_{a b c}^{C}\right]_{L} \times\left[V_{a b c}^{L}\right]
$$

Substituindo (10) na primeira equação do sistema (9) e rearranjando os termos da equação resultante, tem-se:

$$
\left[V_{a b c}^{L}\right]=\left\{\left[A_{(x)}\right]+\left[B_{(x)}\right] \times\left[Y_{a b c}^{C}\right]_{L}\right\}^{-1} \times\left[V_{a b c}^{R}\right]
$$

Substituindo (10) e (11) na segunda equação do sistema (9), tem-se (12), que descreve a relação entre tensões e correntes no terminal remoto através de uma matriz equivalente de admitâncias.

$$
-\left[I_{a b c}^{R}\right]=\left[Y_{a b c}^{e q}\right]_{x} \times\left[V_{a b c}^{R}\right]
$$

Onde:

$$
\begin{aligned}
{\left[Y_{a b c}^{e q}\right]_{x}=\left\{\left[C_{(x)}\right]+\left[D_{(x)}\right] \times\left[Y_{a b c}^{C}\right]_{L}\right\} } \\
\quad \times\left\{\left[A_{(x)}\right]+\left[B_{(x)}\right] \times\left[Y_{a b c}^{C}\right]_{L}\right\}^{-1}
\end{aligned}
$$




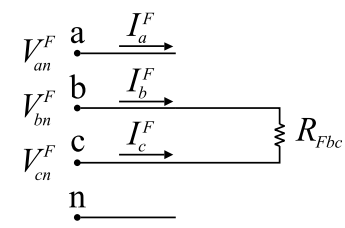

Figura 5: Curto-circuito dupla-fase - BC

\subsection{Curtos-circuitos}

A modelagem dos curtos-circuitos pode ser efetuada por meio de resistências posicionadas de forma conveniente, respeitando o tipo de curto-circuito, as fases envolvidas e o ponto de ocorrência da falta. Sendo assim, os curtos-circuitos podem ser matematicamente representados por uma equação matricial, que relaciona tensões de fase no ponto de falta e as correntes de falta, conforme (14).

$$
\left[I_{a b c}^{F}\right]=\left[Y_{a b c}^{f a l t a}\right] \times\left[V_{a b c}^{F}\right]
$$

Onde:

$\left[Y_{a b c}^{f a l t a}\right]:$ matriz de admitâncias da falta;

$\left[V_{a b c}^{F}\right]:$ tensões de fase no ponto de falta;

$\left[I_{a b c}^{F}\right]$ : correntes de falta.

A título de exemplo, para o curto-circuito dupla-fase (BC) ilustrado em 5, a matriz de admitâncias da falta é dada por (15). Para os outros tipos de curtos-circuitos o procedimento para determinação desta matriz é o mesmo.

$$
\left[Y_{a b c}^{f a l t a}\right]=\left[\begin{array}{ccc}
0 & 0 & 0 \\
0 & R_{F b c}^{-1} & -R_{F b c}^{-1} \\
0 & -R_{F b c}^{-1} & R_{F b c}^{-1}
\end{array}\right]
$$

\section{METODOLOGIA PARA LOCALIZAÇÃO DE FALTAS}

A metodologia proposta neste trabalho foi desenvolvida de acordo com os requisitos descritos no item 2. A identificação do ponto de ocorrência do defeito é efetuada através de quatro etapas que serão descritas a seguir, utilizando como exemplo a rede ilustrada na Fig. 6.

Esta rede é composta por uma linha de transmissão de circuito simples, que interliga os terminais $T_{1}$ e $T_{2}$, de propriedade da concessionária de transmissão. Ao longo do tronco partem três derivações $\left(T_{S 1}, T_{S 2}\right.$ e $\left.T_{S 3}\right)$. Há registros de oscilografia dos sinais de tensões e correntes, de pré e pós-falta,

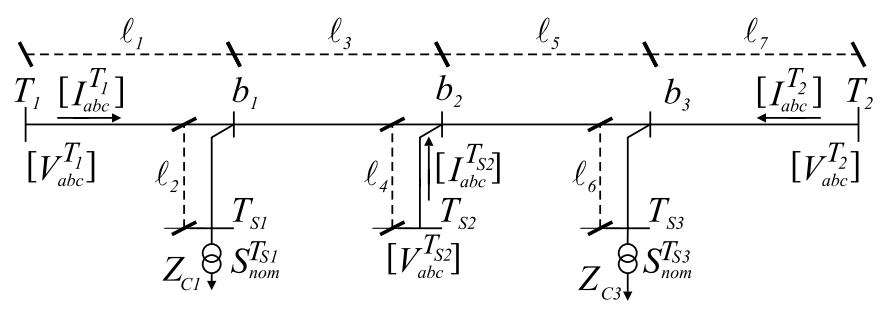

Figura 6: Linha de transmissão

nos terminais $T_{1}$ e $T_{2}$ e apenas na derivação $T_{S 2}$. Estes registros não estão sincronizadas no tempo e não há medições disponíveis nos terminais $T_{S 1}$ e $T_{S 3}$.

\subsection{Processamento dos registros de os- cilografia}

Consiste no processamento digital de sinais para a determinação dos fasores de tensões e correntes nos terminais onde há medições. Para tanto, é necessário filtrar a componente exponencial amortecida presente nestes sinais (de Santana, 1998), para depois efetuar o cálculo dos fasores pelo método da Transformada Discreta de Fourier com janela de um ciclo.

\subsection{Processamento das informações de pré-falta}

O processamento das informações de pré-falta consiste na sincronização entre os registros de tensões e correntes nos terminais onde há medições e na estimativa das impedâncias das cargas nos terminais onde não há medições.

No exemplo apresentado, não há sincronização entre os fasores calculados em $T_{1}, T_{2}$ e $T_{S 2}$ e, para que seja possível efetuar a sincronização entre estes fasores, é necessário estimar os valores das impedâncias das cargas conectadas nos terminais $T_{S 1}$ e $T_{S 3}$ onde não há medições.

Este cálculo é efetuado através do procedimento iterativo ilustrado na Fig. 7 e está dividido nos passos descritos a seguir.

\subsubsection{Cálculo das potências injetadas}

Inicialmente, o algoritmo seleciona o terminal de referência (por exemplo: $T_{1}$ ) e calcula as potências ativa e reativa de pré-falta injetadas na linha $\left(P_{\text {pre }}\right.$ e $\left.Q_{\text {pre }}\right)$, a partir dos fasores de pré-falta das tensões e correntes nos terminais onde há medições $\left(T_{1}, T_{2}\right.$ e $\left.T_{S 2}\right)$.

Essa potências correspondem à somatória das perdas na linha de transmissão e das potências consumidas nos terminais $T_{S 1}$ 


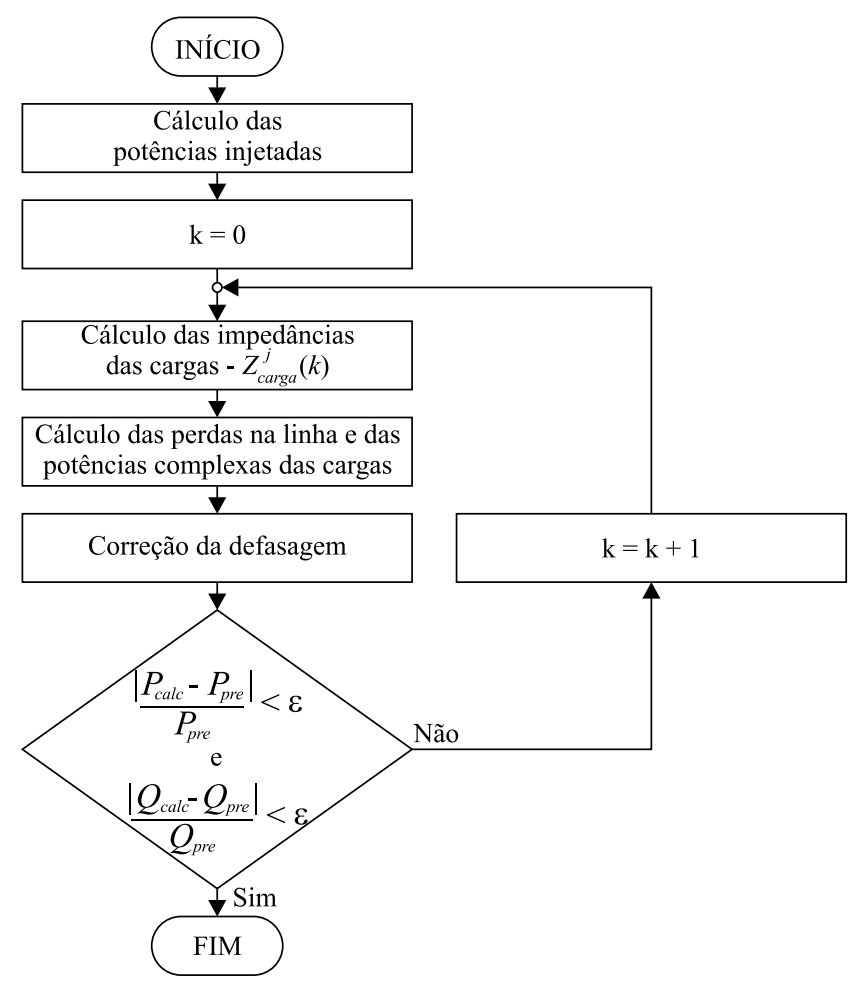

Figura 7: Processamento das informações de pré-falta

e $T_{S 3}$.

\subsubsection{Estimativa das impedâncias das cargas}

O algoritmo estima os valores iniciais das impedâncias nos terminais onde não há medições, a partir das tensões nominais de fase nesses terminais e da distribuição das potências injetadas na linha de forma proporcional à potência nominal dos respectivos transformadores, conforme as equações (16) e (17).

$$
\begin{gathered}
P_{c}^{j}=P_{p r e} \cdot \frac{S_{n}^{j}}{\sum_{j=T_{S 1}}^{T_{S 3}} S_{n}^{j}} \text { e } Q_{c}^{j}=Q_{\text {pre }} \cdot \frac{S_{n}^{j}}{\sum_{j=T_{S 1}}^{T_{S 3}} S_{n}^{j}} \\
Z_{c}^{j}=\frac{3 \cdot\left(V_{n}^{j}\right)^{2}}{\left(P_{c}^{j}+j \cdot Q_{c}^{j}\right)^{*}}
\end{gathered}
$$

Onde:

$S_{n}^{j}$ : potência nominal do transformador em $T_{j}$;

$P_{c}^{j}$ : potência ativa de pré-falta no terminal $T_{j}$;

$Q_{c}^{j}$ : potência reativa de pré-falta no terminal $T_{j}$;
$Z_{c}^{j}$ : estimativa inicial da impedância de carga em $T_{j}$;

$V_{n}^{j}$ : tensão nominal de fase no terminal $T_{j}$.

\subsubsection{Cálculo das perdas na linha e das potências complexas das cargas}

A partir da estimativa das impedâncias das cargas e dos fasores de pré-falta no terminal de referência, é possível determinar as perdas nos trechos da linha, as perdas nos trechos das derivações, as defasagens nos terminais onde há medições e as potências complexas consumidas pelas cargas nos terminais onde não há medições.

Para exemplificar este passo, considere o sistema ilustrado na Fig. 6, cujo terminal de referência é $T_{1}$. A partir da equação (1) é possível calcular os fasores das tensões e correntes de pré-falta no ponto de derivação $b_{1}$ e as perdas no trecho $T_{1}-b_{1}$, utilizando os fasores das tensões e correntes de préfalta em $T_{1}$. Além disso, a partir da equação (12) é possível obter as perdas no trecho $b_{1}-T_{S 1}$ e a potência complexa consumida pela carga em $T_{S 1}$.

Este procedimento permite determinar sucessivamente as perdas em todos os trechos da linha de transmissão e as potências complexas consumidas pelas cargas em todos os terminais onde não há medições $\left(P_{c a l c}\right.$ e $\left.Q_{\text {calc }}\right)$.

\subsubsection{Correção da defasagem}

Conforme descrito no item anterior é possível calcular os fasores das tensões e correntes de pré-falta nos terminais onde há medições, a partir do terminal de referência, utilizando as equações (1) e (12). A correção da defasagem consiste na comparação entre os ângulos destes fasores e os ângulos dos fasores obtidos por meio dos registros efetuados nos terminais onde há medições, conforme descrito em (Manassero, 2006).

\subsubsection{Correção das impedâncias das cargas}

O Processamento das Informações de Pré-falta é um procedimento iterativo que depende das impedâncias das cargas conectadas nos terminais onde não há medições. Sendo assim, o valor destas impedâncias deve ser corrigido a cada iteração de forma a adequar a potência consumida pelas cargas e as perdas na linha de transmissão à potência total injetada nos terminais onde há medições. Esta correção é efetuada conforme a equação (18).

$$
\begin{aligned}
Y_{c}^{j}(k+1)=\operatorname{Re}\left\{Y_{c}^{j}(k)\right\} & \cdot \frac{P_{\text {pre }}}{P_{\text {calc }}}+ \\
& +j \cdot \operatorname{Im}\left\{Y_{c}^{j}(k)\right\} \cdot \frac{Q_{\text {pre }}}{Q_{\text {calc }}}
\end{aligned}
$$


Onde:

$Y_{c}^{j}(k+1)$ : admitância da carga em $T_{j}$;

$k$ : número da iteração.

\subsection{Procedimento para localização da falta}

O procedimento para localização da falta consiste inicialmente no cálculo das tensões e correntes de pós-falta em todos os pontos de derivação, a partir dos fasores das tensões e correntes de pós-falta no terminal $T_{1}$ e no terminal $T_{2}$, resultando em dois grupos de tensões e correntes. Este cálculo depende das impedâncias das cargas nos terminais onde não há medições, dos fasores das tensões e correntes de pós-falta nos terminais onde há medições e é efetuado utilizando as equações (1) e (12), sem considerar a presença da falta no sistema.

Utilizando este procedimento inicial é possível determinar se a falta ocorreu no tronco ou em alguma derivação do sistema de transmissão. Isto é:

- Caso a falta tenha ocorrido em alguma derivação, os fasores das tensões de pós-falta no respectivo ponto de derivação, calculados a partir dos fasores das tensões e correntes de pós-falta nos dois terminais de propriedade da concessionária de transmissão, devem ser iguais. Nesta situação deve-se investigar apenas a derivação em questão;

- Caso contrário, pode-se afirmar que a falta tenha ocorrido em algum trecho pertencente ao tronco. Nesta situação deve-se investigar todos os trechos pertencentes ao tronco do sistema de transmissão.

Para tanto, foram desenvolvidos dois algoritmos. O primeiro, descrito no item 4.4, utiliza os fasores de tensões e correntes de pós-falta em ambos os terminais do trecho investigado. Portanto, é empregado para a localização de faltas nos trechos que pertencem ao tronco, e na derivação onde há medições de tensões e correntes no terminal da respectiva subestação. O segundo, descrito no item 4.5, utiliza medições de tensões e correntes em apenas um dos terminais do trecho investigado. Logo, é empregado para a localização de faltas na derivação onde não há medições no terminal da respectiva subestação.

\subsection{Algoritmo para localização - Tensões e correntes em ambos os terminais}

O algoritmo proposto neste item é empregado para a localização de faltas nos trechos do sistema de transmissão onde

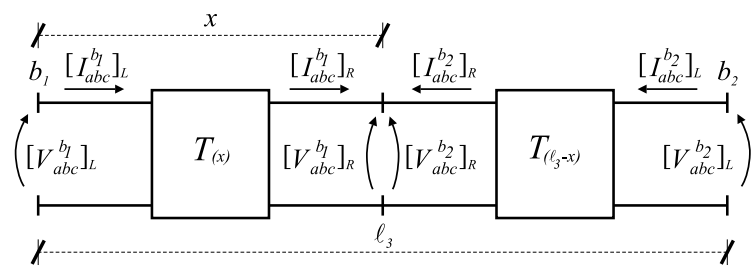

Figura 8: Quadripolos da linha equivalente - Trecho $b_{1}-b_{2}$

os fasores de pós-falta das tensões e correntes são conhecidos em ambos os terminais (Johns and Jamali, 1990).

Para exemplificar este algoritmo, considere o trecho de linha ilustrado em 8. Este trecho possui dois terminais $\left(b_{1}\right.$ e $\left.b_{2}\right)$, comprimento total $l_{3}$ e o ponto de falta se encontra a uma distância desconhecida $x$ de $b_{1}$. Os fasores das tensões e correntes de pós-falta no ponto $b_{1}$ e $b_{2}$ foram calculados a partir dos terminais $T_{1}$ e $T_{2}$, respectivamente, utilizando as equações (1) e (12). Sendo assim, os fasores das tensões e correntes de pós-falta, no ponto de falta podem ser calculadas de acordo com (19) e (20).

$$
\begin{gathered}
{\left[\begin{array}{c}
V_{a b c}^{b_{1}} \\
I_{a b c}^{b_{1}}
\end{array}\right]_{R}=\left[\begin{array}{cc}
{\left[A_{(x)}\right]} & -\left[B_{(x)}\right] \\
-\left[C_{(x)}\right] & {\left[D_{(x)}\right]}
\end{array}\right] \times\left[\begin{array}{c}
V_{a b c}^{b_{1}} \\
I_{a b c}^{b_{1}}
\end{array}\right]_{L}} \\
{\left[\begin{array}{c}
V_{a b c}^{b_{2}} \\
I_{a b c}^{b_{2}}
\end{array}\right]_{R}=\left[\begin{array}{cc}
{\left[A_{\left(l_{3}-x\right)}\right]} & -\left[B_{\left(l_{3}-x\right)}\right] \\
-\left[C_{\left(l_{3}-x\right)}\right] & {\left[D_{\left(l_{3}-x\right)}\right.}
\end{array}\right] \times\left[\begin{array}{c}
V_{a b c}^{b_{2}} \\
I_{a b c}^{b_{2}}
\end{array}\right]_{L}}
\end{gathered}
$$

Para que seja possível estimar o ponto de ocorrência do defeito, é preciso determinar a distância da falta $x$, que torna os fasores das tensões no ponto de falta, calculadas a partir das tensões no ponto de derivação $b_{1}$, iguais às tensões no ponto de falta, calculadas a partir das tensões no ponto de derivação $b_{2}$.

Esta distância pode ser obtida através de um processo de minimização de (21), que representa a diferença entre as tensões no ponto de falta, calculadas a partir de $b_{1}$ e $b_{2}$.

$$
\begin{gathered}
{\left[\Delta V_{a b c}^{f a l t a}\right]=\left[A_{(x)}\right] \times\left[V_{a b c}^{b_{1}}\right]_{L}-\left[B_{(x)}\right] \times\left[I_{a b c}^{b_{1}}\right]_{L}-} \\
-\left[A_{\left(l_{3}-x\right)}\right] \times\left[V_{a b c}^{b_{2}}\right]_{L}+\left[B_{\left(l_{3}-x\right)}\right] \times\left[I_{a b c}^{b_{2}}\right]_{L}
\end{gathered}
$$

É importante ressaltar que o algoritmo proposto depende apenas da diferença entre as tensões no ponto de falta, calculadas a partir dos dois terminais do trecho investigado. Sendo assim, pode-se dizer que o algoritmo é imune a variações na resistência de falta. Além disso, a determinação do ponto de falta pode ser efetuada através de métodos numéricos para minimização de funções, tais como o método da bissecção ou o método da secção áurea. 


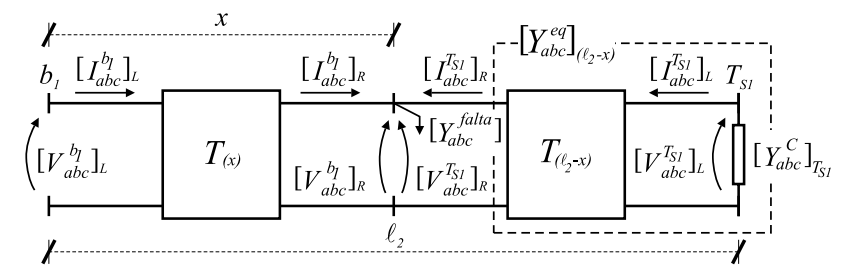

Figura 9: Quadripolos da linha equivalente - Trecho $b_{1}-T_{S 1}$

\subsection{Algoritmo para localização - tensões e correntes em apenas um terminal}

O algoritmo proposto neste item é empregado para a localização de faltas nas derivações do sistema de transmissão, onde os fasores de pós-falta das tensões e correntes são conhecidos apenas no ponto de derivação.

Para exemplificar este algoritmo, considere o trecho de linha ilustrado em 9. Este trecho possui dois terminais $\left(b_{1} \mathrm{e}\right.$ $T_{S 1}$ ), comprimento total $l_{2}$ e o ponto de falta se encontra a uma distância desconhecida $x$ de $b_{1}$. Os fasores das tensões e correntes de pós-falta no ponto $b_{1}$ foram calculados a partir dos terminais $T_{1}$ e $T_{2}$, respectivamente, utilizando as equações (1) e (12). Sendo assim, as tensões e correntes injetadas no ponto de falta podem ser calculadas a partir das tensões e correntes em $b_{1}$, conforme (22).

$$
\left[\begin{array}{c}
V_{a b c}^{b_{1}} \\
I_{a b c}^{b_{1}}
\end{array}\right]_{R}=\left[\begin{array}{cc}
{\left[A_{(x)}\right]} & -\left[B_{(x)}\right] \\
-\left[C_{(x)}\right] & {\left[D_{(x)}\right.}
\end{array}\right] \times\left[\begin{array}{c}
V_{a b c}^{b_{1}} \\
I_{a b c}^{b_{1}}
\end{array}\right]_{L}
$$

E as correntes no ponto de falta conforme (23).

$$
\left[I_{a b c}^{f a l t a}\right]=\left[I_{a b c}^{b_{1}}\right]_{R}+\left[I_{a b c}^{T_{S 1}}\right]_{R}=\left[Y_{a b c}^{f a l t a}\right] \times\left[V_{a b c}^{b_{1}}\right]_{R}
$$

Uma vez que não há medições de tensões e correntes em $T_{S 1}$, não é possível determinar as correntes injetadas no ponto de falta, a partir do quadripolo de transmissão do trecho de linha em questão - trecho $\left(l_{2}-x\right)$. Entretanto, é possível obtê-las através da utilização da matriz de admitâncias da associação entre transformador, carga e trecho de linha, definida no item 3.3 (13). Portanto:

$$
-\left[I_{a b c}^{T_{S 1}}\right]_{R}=\left[Y_{a b c}^{e q}\right]_{\left(l_{2}-x\right)} \times\left[V_{a b c}^{b_{1}}\right]_{R}
$$

Substituindo (24) em (23) tem-se:

$$
\left(\left[Y_{a b c}^{f a l t a}\right]+\left[Y_{a b c}^{e q}\right]_{\left(l_{2}-x\right)}\right) \times\left[V_{a b c}^{b_{1}}\right]_{R}=\left[I_{a b c}^{b_{1}}\right]_{R}
$$

Para a determinação da distância $x$ é preciso utilizar os fasores das tensões e correntes em $b_{1}$. Portanto, substituindo (22) em (25), é possível obter (26) que relaciona as tensões e correntes na entrada do quadripolo $T_{(x)}$ por meio dos parâmetros deste quadripolo, das matrizes de admitâncias de falta e da associação carga, transformador e trecho de linha.

$$
\left[V_{a b c}^{b_{1}}\right]_{L}=\left[Z_{e q}\right] \times\left[I_{a b c}^{b_{1}}\right]_{L}
$$

Onde:

$$
\begin{aligned}
& {\left[Z_{e q}\right]=\left\{\left[A_{(x)}\right]+\left[B_{(x)}\right] \times\left(\left[Y_{a b c}^{\text {falta }}\right]+\left[Y_{a b c}^{e q}\right]_{\left(l_{2}-x\right)}\right)\right\} \times} \\
& \times\left\{\left[C_{(x)}\right]+\left[D_{(x)}\right] \times\left(\left[Y_{a b c}^{\text {falta }}\right]+\left[Y_{a b c}^{e q}\right]_{\left(l_{2}-x\right)}\right)\right\}^{-1}
\end{aligned}
$$

Uma vez que as impedâncias das cargas foram estimadas na etapa de processamento das informações de pré-falta, a matriz de impedâncias equivalente $\left[Z_{e q}\right]$ é função apenas da distância $x$ e dos elementos da matriz de admitâncias da falta, conforme (28).

$$
\left[Z_{e q}\right]=f\left(x,\left[Y_{a b c}^{f a l t a}\right]\right)
$$

Desta forma, o procedimento para localização da falta consiste em determinar a distância $x$ e as impedâncias de falta da matriz de impedâncias equivalente, que satisfazem o sistema (26). Para tanto, o algoritmo proposto neste item emprega o método de Newton-Raphson.

Para cada tipo de falta há uma matriz $\left[Z_{e q}\right]$ diferente e, portanto, equações diferentes para a determinação do ponto de mínimo do sistema (26). Neste trabalho está apresentado apenas o desenvolvimento da equação para um curto-circuito fase-terra (AN), para outros tipos de curto-ciruito basta proceder da forma descrita.

Para o curto-circuito fase-terra a matriz $\left[Z_{e q}\right]$ é função da distância $x$ e do elemento da matriz de admitâncias da falta $Y_{F a}$, conforme a equação 29 .

$$
\left[Z_{e q}\right]=f\left(x, Y_{F a}\right)
$$

Para determinar $x$ e $Y_{F a}$, o algoritmo proposto parte de um valor inicial para ambas as variáveis. A cada iteração, estes valores são corrigidos conforme apresentado em 31 .

$$
\begin{gathered}
x(k+1)=x(k)+\Delta x(k) \\
Y_{F a}(k+1)=Y_{F a}(k)+\Delta Y_{F a}(k)
\end{gathered}
$$

Onde $k$ é o número da iteração e $\Delta x(k)$ e $\Delta Y_{F a}(k)$ podem ser obtidos através da solução da equação 32.

$$
\begin{aligned}
& {\left[V_{a b c}\right]_{L}-\left[Z_{e q}\right]_{(k)} \times\left[I_{a b c}\right]_{L}=\left[\Delta V_{a b c}^{b_{1}}\right]_{k}=} \\
& \left\{\frac{\partial\left[Z_{e q}\right]_{(k)}}{\partial x} \Delta x(k)+\frac{\partial\left[Z_{e q}\right]_{(k)}}{\partial Y_{F a}} \Delta Y_{F a}(k)\right\} \times\left[I_{a b c}\right]_{L}
\end{aligned}
$$


Uma vez que o número de equações é superior ao número de incógnitas (seis equações - três referentes à parte real e três referentes à parte imaginária da equação 32 e apenas duas incógnitas) é necessário utilizar um método para a regressão linear do sistema. O algoritmo proposto neste trabalho utiliza o método dos mínimos quadrados e está detalhado na referência (Manassero, 2006).

\subsection{Determinação do trecho de ocorrên- cia da falta}

O sistema para localização de faltas proposto neste trabalho indica dois possíveis pontos de falta. Um deles pertencente ao tronco do sistema de transmissão e outro pertencente à derivação investigada.

É importante ressaltar que a investigação dos trechos pertencentes ao tronco resulta em um ponto de falta para cada trecho investigado. A cada ponto está associado o valor de mínimo da função descrita pela equação 21. Caso a falta tenha ocorrido no tronco, o valor mínimo desta função deve ser zero, no trecho onde a falta ocorreu. Portanto, após a investigação de todos os trechos, apenas um se torna o possível candidato (aquele que possui o menor valor de mínimo da função).

\section{SIMULAÇÕES DA METODOLOGIA PARA LOCALIZAÇÃO DE FALTAS}

A rede de transmissão utilizada para testes foi implementada no ATP e está ilustrada em 10. Esta rede é composta por uma linha de transmissão de 138 [kV], de circuito duplo, com cinco derivações. No terminal $T_{1}$ há uma fonte de tensão trifásica ideal em série com uma matriz de impedâncias equivalentes, que representam as potências de curto-circuito trifásica e monofásica neste terminal. $\mathrm{O}$ mesmo ocorre para o terminal $T_{2}$.

A torre e os condutores escolhidos para montagem dos casos de simulação são utilizados em linhas de transmissão típicas, de circuito duplo e nível de tensão de 138 [kV]. A linha de transmissão utilizada também foi implementada no software ATP, através da rotina LINE CONSTANTS. O tipo de torre utilizado na montagem dos casos de simulação está ilustrado em 11.

A linha de transmissão é composta por condutores de fase do tipo CAA (alumínio com alma de aço), bitola de 266,8 MCM (Partridge), cujas flechas têm 6,5 [m] e cabo pára-raios constituído por cabos de aço galvanizado (7 fios) tipo HS, diâmetro nominal de $1 / 2$ polegada, cuja flecha é 6,0 [m]. A linha não é transposta e a resistividade do solo é 400 [ohms.m].

A tabela 1 apresenta uma descrição dos casos de simulação

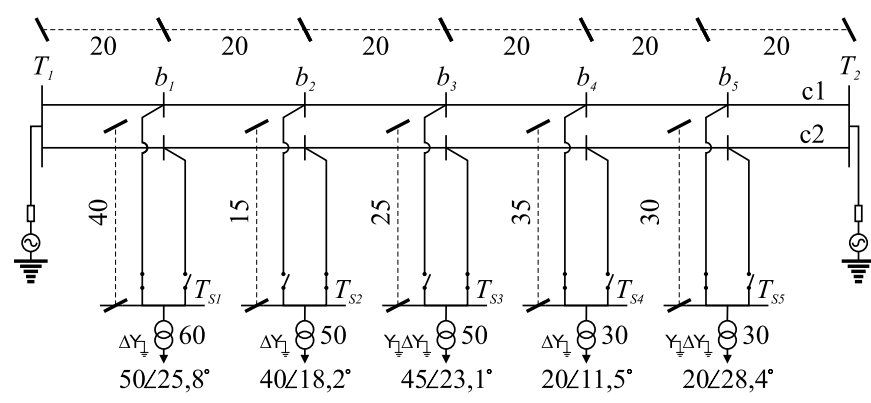

Notas:

1) As potências das cargas e dos transformadores estão em [MVA]

2) Potências de curto-circuito $-T_{1} \rightarrow 3 \phi: 1993 \angle 74,74^{\circ}$ e $1 \phi: 1518 \angle 78,44^{\circ}$

2) Potências de curto-circuito $-T_{2} \rightarrow 3 \phi: 3899 \angle 86,13^{\circ}$ e $1 \phi: 2927 \angle 87,06^{\circ}$

3) Os comprimentos dos trechos de linha estão em [km]

Figura 10: Modelo simplificado do sistema implementado no ATP

implementados no ATP. Foram simulados curtos-circuitos fase-terra, dupla-fase, dupla-fase-terra e trifásico em vinte e dois pontos distintos da rede, com diversas resistências de falta. Todas as simulações possuem cinco ciclos e foram utilizados dois instantes de ocorrência da falta distintos (após 2 ciclos e $2 \frac{1}{4}$ ciclos de simulação), para se verificar a influência da componente exponencial amortecida nos sinais de corrente. Como resultado da variação desses parâmetros durante a montagem dos arquivos-fonte do ATP, foi possível

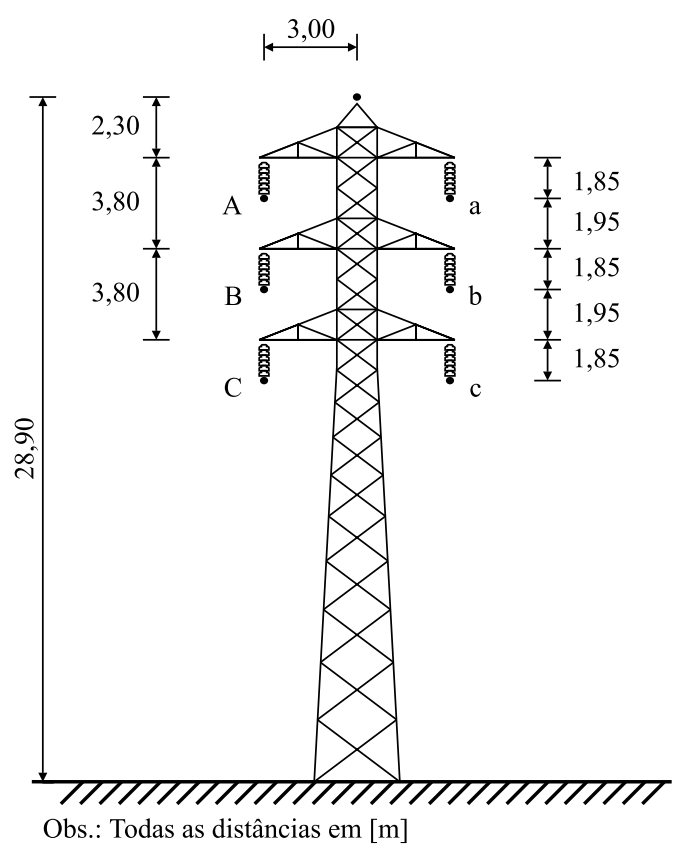

Figura 11: Tipo de torre utilizada nas simulações 
Tabela 1: Casos de simulação do ATP.

\begin{tabular}{|c|c|c|c|c|}
\hline $\begin{array}{l}\text { Tipo de } \\
\text { falta }\end{array}$ & $\begin{array}{c}\text { Instante } \\
{[\mathrm{ms}]}\end{array}$ & $\begin{array}{l}\text { No. de } \\
\text { pontos }\end{array}$ & $\begin{array}{l}R_{F} \\
{[\Omega]}\end{array}$ & $\begin{array}{c}\text { Total de } \\
\text { casos }\end{array}$ \\
\hline AN & $\begin{array}{l}33,33 \\
37,50\end{array}$ & 22 & $\begin{array}{c}0,1,5,20 \\
50 \text { e } 100\end{array}$ & 264 \\
\hline $\begin{array}{c}\mathrm{BC} \\
\mathrm{BCN}\end{array}$ & 33,33 & 22 & 0,1 e 5 & 132 \\
\hline $\mathrm{ABC}$ & 33,33 & 22 & 0 e 1 & 44 \\
\hline
\end{tabular}

obter um total de 440 casos de simulação.

\section{RESULTADOS}

O sistema proposto neste trabalho foi submetido a testes para avaliação do seu desempenho frente aos casos de simulação descritos no item anterior. Para a avaliação da precisão da metodologia proposta, foram calculados os erros em módulo, na distância do ponto de falta até um dos terminais do trecho sob investigação, através da equação 33 .

$$
\epsilon_{D}=\left|D_{\text {Calculada }}-D_{\text {Correta }}\right|
$$

Os resultados obtidos estão apresentados nos itens a seguir.

\subsection{Medição em todos os terminais}

A tabela 2 apresenta os erros mínimo, máximo e médio no cálculo da distância da falta considerando faltas fase-terra, com resistências de falta de $0,1,5,20$ e $50[\Omega]$; faltas duplafase, com resistências de falta de 0,1 e $5[\Omega]$; dupla-faseterra, com resistências de falta de 0,1 e $5[\Omega]$; e faltas trifásicas, com resistências de falta de 0 e $1[\Omega]$.

Conforme detalhado nesta tabela, os erros máximos são inferiores a $440[\mathrm{~m}]$ para todos os tipos de falta. Os erros médios para as faltas dupla-fase, dupla-fase-terra e trifásicas são aproximadamente iguais. Para a falta fase-terra o erro médio é um pouco mais elevado devido às resistência de falta elevadas $(50[\Omega])$. Entretanto, estes erros podem ser considerados pequenos quando comparados ao comprimento total da linha

Tabela 2: Medições em todos os terminais.

\begin{tabular}{ccccc}
\hline \hline $\begin{array}{c}\text { Tipo de } \\
\text { Falta }\end{array}$ & mín & $\begin{array}{c}\epsilon_{D}[\mathbf{m}] \\
\text { máx }\end{array}$ & médio & $\begin{array}{c}\text { Desvio } \\
\text { padrão [m] }\end{array}$ \\
\hline $\mathrm{AN}$ & 0 & 432 & 111 & 96 \\
$\mathrm{BC}$ & 3 & 248 & 93 & 80 \\
$\mathrm{BCN}$ & 1 & 246 & 80 & 72 \\
$\mathrm{ABC}$ & 1 & 288 & 52 & 48 \\
\hline \hline
\end{tabular}

Tabela 3: Medições apenas em $T_{1}$ e $T_{2}$.

\begin{tabular}{ccccc}
\hline \hline $\begin{array}{c}\text { Tipo de } \\
\text { Falta }\end{array}$ & mín & $\begin{array}{c}\epsilon_{D}[\mathbf{m}] \\
\text { máx }\end{array}$ & $\begin{array}{c}\text { Desvio } \\
\text { médio }\end{array}$ & $\begin{array}{c}\text { padrão [m] } \\
\text { padgoritmo descrito no item }\end{array}$ \\
\hline \multicolumn{5}{c}{ Alg.4 } \\
\hline AN & 1 & 585 & 168 & 128 \\
BC & 0 & 44 & 18 & 12 \\
BCN & 0 & 52 & 19 & 16 \\
ABC & 0 & 132 & 38 & 37 \\
\hline \multicolumn{5}{c}{ Algoritmo descrito no item 4.5 } \\
\hline AN & 1 & 1708 & 290 & 379 \\
BC & 84 & 1029 & 305 & 260 \\
BCN & 78 & 1042 & 290 & 271 \\
ABC & 1 & 848 & 175 & 248 \\
\hline \hline
\end{tabular}

de transmissão.

\subsection{Medição nos terminais $T_{1}$ e $T_{2}$}

A tabela 3 apresenta os erros mínimo, máximo e médio no cálculo da distância da falta para o algoritmos propostos nos itens 4.4 e 4.5 considerando faltas fase-terra, com resistências de falta de $0,1,5,20$ e $50[\Omega]$; faltas dupla-fase, com resistências de falta de 0,1 e $5[\Omega]$; dupla-fase-terra, com resistências de falta de 0,1 e $5[\Omega]$; e faltas trifásicas, com resistências de falta de 0 e $1[\Omega]$.

O algoritmo descrito no item 4.4 apresentou erros máximos inferiores a $600[\mathrm{~m}]$ para faltas fase-terra, inferiores a 55 [m] para faltas dupla-fase e dupla-fase-terra, e inferiores a 135 [m] para faltas trifásicas. O algoritmo descrito no item 4.5 apresentou erros máximos inferiores a 1710 [m] para faltas fase-terra, inferiores a $1050[\mathrm{~m}]$ para os curtos-circuitos dupla-fase e dupla-fase-terra e inferiores a 900 [m] para os curtos-circuitos trifásicos. Estes erros podem ser considerados pequenos quando comparados ao comprimento total da linha de transmissão.

\subsection{Influência da resistência de falta}

Para análise da influência da resistência de falta no desempenho dos algoritmos propostos, foram simulados curtoscircuitos fase-terra - AN, com resistências de falta variando de 0 a 100 ohms. A tabela 4 apresenta os erros no cálculo da distância da falta.

O efeito da resistência de falta no desempenho do algoritmo descrito no item 4.4 pode ser observado em 12. Esta figura ilustra o valor da função descrita pela equação 21 no trecho da linha de transmissão que se encontra em falta e, conforme apresentado no item 4.4, o ponto de falta encontra-se no ponto de mínimo dessa função. 
No gráfico (a), as impedâncias das cargas das subestações em derivação são conhecidas. Neste caso, pode-se notar que o ponto de mínimo independe do valor das resistências de falta. No gráfico (b) as impedâncias das cargas foram estimadas conforme apresentado no item 4.2.2. Neste caso, pode-se notar que o ponto de mínimo da função se desloca à medida que aumenta a resistência de falta. A partir da comparação entre ambos os gráficos pode-se afirmar que o aumento no erro decorre da imprecisão na estimativa das impedâncias das cargas, e não do algoritmo de localização de faltas.

\subsection{Infuência do modelo da linha de transmissão}

Os algoritmos propostos neste trabalho, têm acesso aos parâmetros elétricos dos trechos de linha que compõem a rede de transmissão. Para a análise da influência da variação nos parâmetros desses trechos e do modelo de linha empregado para representá-los, foram alterados esses parâmetros, ao invés de alterar os casos de simulação no ATP.

Inicialmente, foi feita uma análise da influência da modelagem correta da transposição da linha no cálculo da distância. Para tanto, os algoritmos de localização utilizaram dados de uma linha de transmissão idealmente transposta.

Em seguida, foi feita uma análise da influência das capacitâncias da linha no cálculo da distância. Para esta análise, os algoritmos de localização utilizaram apenas a matriz de impedâncias série da linha de transmissão.

Para finalizar, foi feita uma análise da influência da variação dos parâmetros da linha de transmissão no cálculo da distân-

Tabela 4: Variações na resistência de falta.

\begin{tabular}{ccccc}
\hline \hline $\begin{array}{l}\text { Resistência } \\
\text { de falta }[\Omega]\end{array}$ & mín & $\begin{array}{c}\epsilon_{D}[m] \\
\text { máx }\end{array}$ & $\begin{array}{c}\text { Desvio } \\
\text { médio }\end{array}$ & $\begin{array}{c}\text { Desão } \\
\text { padão }\end{array}$ \\
\hline \multicolumn{5}{c}{ Algoritmo descrito no item 4.4} \\
\hline 0 & 12 & 223 & 113 & 67 \\
1 & 10 & 223 & 114 & 68 \\
5 & 1 & 233 & 119 & 73 \\
20 & 16 & 349 & 180 & 104 \\
50 & 23 & 585 & 315 & 168 \\
100 & 17 & 932 & 527 & 288 \\
\hline \multicolumn{5}{c}{ Algoritmo descrito no item 4.5} \\
\hline 0 & 6 & 810 & 143 & 232 \\
1 & 3 & 825 & 147 & 238 \\
5 & 22 & 884 & 196 & 248 \\
20 & 3 & 1148 & 327 & 323 \\
50 & 1 & 1708 & 639 & 539 \\
100 & 233 & 3063 & 1558 & 990 \\
\hline \hline
\end{tabular}

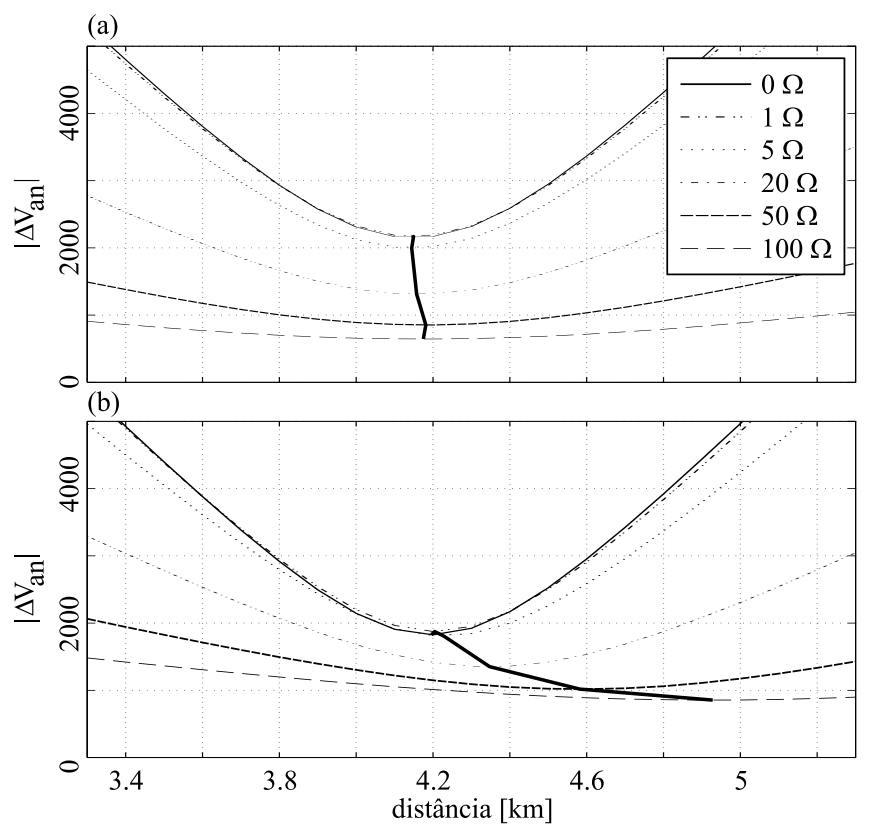

Figura 12: Valor da função descrita pela equação 21

cia. Para tanto, os algoritmos de localização utilizaram dados de uma linha de transmissão que possui uma flecha $10 \%$ maior que a flecha utilizada nas simulações do ATP e uma resistividade de 1200 [ohms.m] ao invés de 400 [ohms.m].

Conforme detalhado na tabela 5, o algoritmo descrito no item 4.4 apresenta erros máximos inferiores a 200 metros quando se utiliza os parâmetros corretos da linha de transmissão. Os erros são um pouco mais elevados quando se utiliza a matriz de impedâncias série para representar a linha e tornam-se ainda maiores quando se utiliza os parâmetros incorretos.

O algoritmo descrito no item 4.5 apresenta erros máximos inferiores a 370 metros quando se utiliza os parâmetros corretos da linha de transmissão. Os erros são um pouco mais elevados quando se utiliza a matriz de impedâncias série para representar a linha e tornam-se ainda maiores quando se utiliza os parâmetros incorretos.

\section{CONCLUSÕES}

Este trabalho apresentou o desenvolvimento de um sistema para localização de faltas em linhas de transmissão com subestações conectadas em derivação e, mais especificamente, os algoritmos que integram o sistema proposto. Os resultados apresentados indicam que os algoritmos propostos possuem um nível de precisão adequado, uma vez que o erro máximo encontrado para o algoritmo descrito no item 4.4 é 
Tabela 5: Variações nos parâmetros das linhas de transmissão.

\begin{tabular}{|c|c|c|c|c|}
\hline $\begin{array}{c}\text { Tipo } \\
\text { de falta }[\Omega]\end{array}$ & mín & $\begin{array}{l}\epsilon_{D}[m] \\
\text { máx }\end{array}$ & médio & $\begin{array}{l}\text { Desvio } \\
\text { padrão }\end{array}$ \\
\hline \multicolumn{5}{|c|}{ Algoritmo descrito no item 4.4} \\
\hline \multicolumn{5}{|c|}{ Parâmetros corretos e carga ideal } \\
\hline $\mathrm{AN}$ & 2 & 172 & 77 & 48 \\
\hline $\mathrm{BC}$ & 1 & 75 & 28 & 21 \\
\hline $\mathrm{BCN}$ & 3 & 62 & 25 & 16 \\
\hline $\mathrm{ABC}$ & 6 & 91 & 31 & 27 \\
\hline
\end{tabular}

Linha de transmissão idealmente transposta e carga ideal

\begin{tabular}{|c|c|c|c|c|}
\hline AN & 2 & 91 & 40 & 23 \\
\hline $\mathrm{BC}$ & 0 & 143 & 58 & 43 \\
\hline $\mathrm{BCN}$ & 7 & 210 & 85 & 60 \\
\hline $\mathrm{ABC}$ & 2 & 318 & 128 & 92 \\
\hline \multicolumn{5}{|c|}{ Modelo da linha sem capacitâncias e carga ideal } \\
\hline AN & 0 & 221 & 86 & 50 \\
\hline $\mathrm{BC}$ & 15 & 339 & 198 & 96 \\
\hline $\mathrm{BCN}$ & 23 & 315 & 197 & 85 \\
\hline $\mathrm{ABC}$ & 47 & 316 & 182 & 74 \\
\hline \multicolumn{5}{|c|}{ Parâmetros incorretos e carga ideal } \\
\hline AN & 1 & 633 & 300 & 196 \\
\hline $\mathrm{BC}$ & 1 & 201 & 82 & 57 \\
\hline $\mathrm{BCN}$ & 3 & 191 & 78 & 52 \\
\hline $\mathrm{ABC}$ & 1 & 144 & 62 & 50 \\
\hline \multicolumn{5}{|c|}{ Algoritmo descrito no item 4.5} \\
\hline \multicolumn{5}{|c|}{$\overline{\text { Parâmetros corretos e carga ideal }}$} \\
\hline AN & 3 & 369 & 116 & 76 \\
\hline $\mathrm{BC}$ & 130 & 349 & 238 & 61 \\
\hline $\mathrm{BCN}$ & 109 & 352 & 221 & 64 \\
\hline $\mathrm{ABC}$ & 54 & 151 & 106 & 27 \\
\hline \multicolumn{5}{|c|}{ Linha de transmissão idealmente transposta e carga ideal } \\
\hline $\mathrm{AN}$ & 16 & 867 & 205 & 188 \\
\hline $\mathrm{BC}$ & 719 & 1885 & 1282 & 376 \\
\hline $\mathrm{BCN}$ & 68 & 2731 & 1082 & 1489 \\
\hline $\mathrm{ABC}$ & 56 & 268 & 150 & 69 \\
\hline \multicolumn{5}{|c|}{ Modelo de linha sem capacitâncias e carga ideal } \\
\hline AN & 140 & 1034 & 470 & 243 \\
\hline $\mathrm{BC}$ & 207 & 1067 & 530 & 259 \\
\hline $\mathrm{BCN}$ & 141 & 1052 & 487 & 263 \\
\hline $\mathrm{ABC}$ & 198 & 897 & 445 & 230 \\
\hline \multicolumn{5}{|c|}{ Parâmetros incorretos e carga ideal } \\
\hline AN & 468 & 1758 & 939 & 382 \\
\hline $\mathrm{BC}$ & 397 & 956 & 665 & 169 \\
\hline $\mathrm{BCN}$ & 132 & 728 & 294 & 142 \\
\hline $\mathrm{ABC}$ & 314 & 726 & 528 & 126 \\
\hline
\end{tabular}

inferior a 1000 metros e o erro máximo encontrado para o algoritmo descrito no item 4.5 é inferior a 3100 metros $(0,4 \%$ e $1,2 \%$ do comprimento total da linha de transmissão, respec- tivamente).

Conforme apresentado no item 6, pode-se concluir que o desempenho da metodologia proposta neste trabalho depende do nível de detalhamento dos modelos utilizados e da quantidade de informação disponível para a localização da falta. Desta forma, os resultados apresentados no item 6.1 possuem um nível de precisão maior que os resultados apresentados no item 6.2. Isto ocorre porque no item 6.1 há medições de tensões e correntes em todos terminais e no item 6.2 há medições em apenas dois terminais.

Além disso, é importante modelar corretamente as linhas de transmissão e estimar corretamente as cargas, para que a precisão dos algoritmos não seja afetada. No item 6.3 pode-se perceber que a estimativa das cargas influencia a localização da falta para curtos-circuitos com resistências de falta elevadas e no item 6.4 fica claro que o modelo empregado para representar a linha de transmissão interfere no desempenho dos algoritmos propostos.

\section{REFERÊNCIAS}

Abe, M., Otsuzuki, N., Emura, T. and Takeuchi, M. (1995). Development of a new fault location system for multiterminal single transmission lines, IEEE Transactions on Power Delivery 10(1): 159-168.

Aggarwal, R. K., Coury, D. V., Johns, A. T. and Kalam, A. (1993). A practical approach to accurate fault location on extra high voltage teed feeders, IEEE Transactions on Power Delivery 8(3): 874-883.

Bowman, W. I. and McNamee, J. M. (1964). Development of equivalent $\mathrm{Pi}$ and $\mathrm{T}$ matrix circuits for long untransposed transmission lines, IEEE Transactions on Power Apparatus and Systems 83(6): 625-632, 653-657.

Brahma, S. M. (2005). Fault location scheme for a multiterminal transmission line using synchronized voltage measurements, IEEE Transactions on Power Delivery 20(2): 1325-1331.

de Santana, N. N. (1998). Pré-filtragem da componente aperiódica exponencial para os algoritmos utilizados em relés digitais de distância, Master's thesis, Escola Politécnica da USP, São Paulo, Brazil.

Funabashi, T., Otoguro, H., Mizuma, Y., Dube, L. and Ametani, A. (2000). Digital fault location for parallel double-circuit multi-terminal transmission lines, IEEE Transactions on Power Delivery 15(2): 531-537.

Girgis, A., Hart, D. and Peterson, W. (1992). A new fault location technique for two and three terminal lines, IEEE Transactions on Power Delivery 7(1): 98-107. 
Johns, A. T. and Jamali, S. (1990). Accurate fault location technique for power transmission lines, IEE Proceedings - Generation, Transmission and Distribution 137, part C(6): 395-402.

Lai, L. L., Rajkumar, N., Vaseekar, E., Subasinghe, H., Carter, A. and Gwyn, B. J. (2000). Wavelet transform and neural networks for fault location of a teed-network, Proceedings of the International Conference on Power System Technology, 2000, PowerCon 2000, Perth, Austrá lia, pp. 807-811. vol. 2.

Lin, Y., Liu, C. and Yu, C. (2002). A new fault locator for three-terminal transmission lines using two-terminal synchronized voltage and current phasors, IEEE Transactions on Power Delivery 17(2): 452-459.

Manassero, Jr., G. (2006). Sistema para Localização de Faltas em Linhas de Transmissão com Subestações Conectadas em Derivação, PhD thesis, Escola Politécnica da USP, São Paulo, Brazil.

Nagasawa, T., Abe, M., Otsuzuki, N., Emura, T., Jikihara, Y. and Takeuchi, M. (1992). Development of a new fault location algorithm for multi-terminal two-parallel transmission lines, IEEE Transactions on Power Delivery 7(3): $1516-1532$.

Ramos, D. S. and Dias, E. M. (1983). Sistemas elétricos de potência - Regime permanente, Vol. 2, Ed. Guanabara Dois, Rio de Janeiro, RJ.

Tziouvaras, A. D., Roberts, J. B. and Benmouyal, G. (2001). New multi-ended fault location design for two- or three-terminal lines, Developments in Power System Protection, 2001, Seventh International Conference on, IEE, Amsterdã, pp. 395-398. no. 479.

Yu, C., Liu, C. and Lin, Y. (2001). A fault location algorithm for transmission lines with tapped leg - PMU based approach, Summer Meeting 2001, IEEE PES, Vancouver, pp. 915-920. 